\title{
Effect of Early Postnatal Body Weight on Femoral Head Ossification Onset and Hip Osteoarthritis in a Canine Model of Developmental Dysplasia of the Hip
}

\author{
WENDY S. VANDEN BERG-FOELS, RORY J. TODHUNTER, STEVEN J. SCHWAGER, AND ANTHONY P. REEVES \\ Department of Biomedical Engineering [W.S.V.B.-F.], Department of Clinical Sciences [R.J.T.], Department of Biological Statistics and \\ Computational Biology [S.J.S.], Department of Electrical and Computer Engineering [A.P.R.], Cornell University, Ithaca, NY 14853
}

\begin{abstract}
Developmental dysplasia of the hip (DDH) is a wellknown precipitator of hip osteoarthritis. An increase in body weight during the critical early postnatal growth period may alter joint contact, and thus alter hip development and influence joint health in adulthood. The objective of this study was to determine whether early postnatal body weight affected the course of hip development and the onset of osteoarthritis in a canine model of DDH. A longitudinal study, from birth to skeletal maturity, was conducted. Serial body weight, age at femoral head ossification onset, and femoral head coverage at 4 mo were measured. Presence and severity of degeneration at 8 mo were determined using necropsy and cartilage biochemistry. There was a negative association between birth weight and age at femoral head ossification onset; however, the association was likely due to skeletal maturity level rather than body weight per se. Lower birth weight subjects had greater femoral head coverage at 4 mo. Greater birth weight was associated with greater probability of moderate degenerative changes or macroscopic lesions at 8 mo. These results support the hypothesis that increased birth weight is sufficient to alter the course of hip development and result in measurable degenerative changes at adulthood. (Pediatr Res 60: 549554,2006 )
\end{abstract}

$\mathrm{D}$ evelopmental dysplasia of the hip (DDH), characterized in the adult by a shallow acetabulum and a flattened femoral head, is a well-known precipitator of early onset hip osteoarthritis (OA) (1-3). In the human neonate (4) and juvenile dog (5), DDH ranges from joint instability to complete dislocation of the femoral head.

Incidence estimates for DDH in humans vary with examination technique, child age, and disease definition; the true incidence is unknown $(1,4)$. Screening surveys suggest that as many as 1 in 100 newborns have evidence of hip instability (4). Twenty percent to $50 \%$ of idiopathic OA cases have been attributed to underlying subluxation or acetabular dysplasia (1). Risk of DDH is greater for first borns, breech position, female gender, left hips, an adverse intrauterine environment such as oligohydramnios, and infants swaddled in a leg-

Received January 10, 2006; accepted June 20, 2006.

Correspondence: Wendy S. Vanden Berg-Foels, 357 Rhodes Hall, Department of Electrical Engineering, Cornell University, Ithaca, NY 14853; email: wsf2@cornell.edu Supported by the New York State Advanced Technology Biotechnology Program (R.J.T., W.S.V.B.-F.), Cornell University College of Veterinary Medicine Consolidated Research Grant Program (R.J.T., W.S.V.B.-F.), NSF and AAUW Graduate Fellowships (W.S.V.B.-F.)

DOI: 10.1203/01.pdr.0000243546.97830.a0 extended position $(1,4)$. Greater risk of left hip DDH is likely a result of two thirds of vertex presentations being in the left occiput position $(1,6)$. Abduction of the left hip is limited as the fetus is pressed against the mother's spine resulting in decreased coverage of the femoral head by the acetabulum (7). Risk is also greater for positive family history (affected parent $12 \%$; affected parent and sibling 36\%) (1).

Incidence estimates for DDH in dogs vary by breed, and are greater for larger breeds. Approximately $20 \%$ of Labrador retrievers, a large breed, are affected; incidence exceeds $50 \%$ for St. Bernards (8). Heritability estimates range from 0.25 to 0.40 (9).

The dog is an established, naturally occurring model for the study of early onset OA secondary to DDH and shares the characteristic morphologic and biochemical features of DDH in humans $(1,5,9)$. A gender difference in incidence has not been demonstrated in the dog model, and incidence is greater for larger breeds than for humans. Dogs are skeletally mature by $8 \mathrm{mo}$, enabling longitudinal observation of DDH from incipience to degeneration.

A critical stage in hip development occurs during the early postnatal period. In humans, the depth of the acetabulum relative to its diameter is a minimum at birth, requiring that the joint capsule and ligament of the femoral head play a greater role in maintaining stability and congruent articulation (7). Although no comparable study exists for dogs, the capsule and ligament of the femoral head are largely responsible for maintaining congruent articulation for the first month after birth (5). Experimental animal models have demonstrated that fully congruent contact between the cartilaginous femoral head and acetabulum (Fig. 1 morphology) is necessary for development of normal joint morphology $(10,11)$ and that the neonatal joint has a remarkable capacity to respond to the local mechanical environment (12). In a rat model with a unilateral amputation below the femoral neck, presence of the femoral head within the acetabulum was sufficient to retain

Abbreviations: DDH, developmental dysplasia of the hip; EGR, exponential growth rate; GAG, glycosaminoglycan; HLM, hierarchical linear model; OA, osteoarthritis 


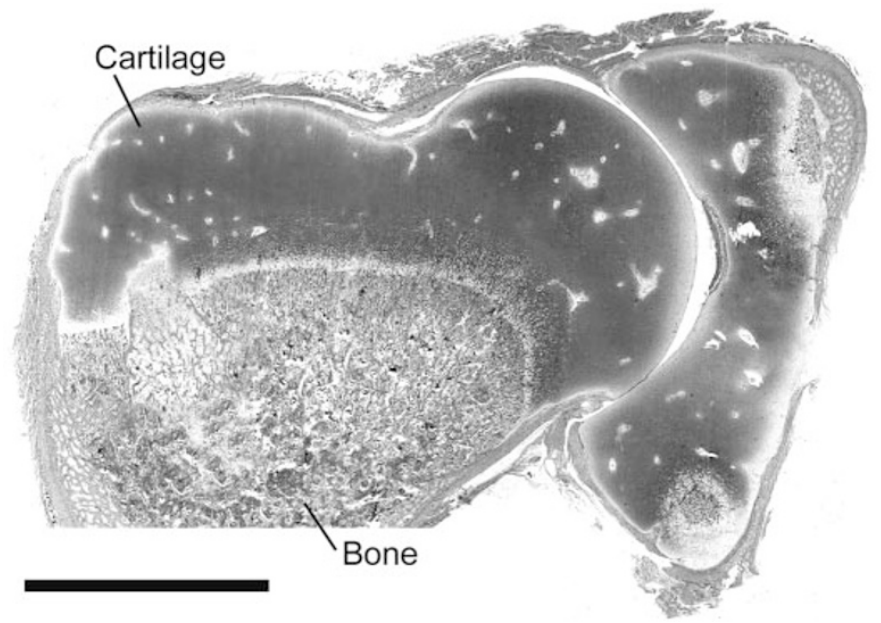

Figure 1. Hip at $1 \mathrm{~d}$ of age. $\mathrm{Bar}=5 \mathrm{~mm}$.

congruent articulating surfaces (11). The rate of growth relative to size is at a maximum during the early postnatal period (Fig. 2). Small perturbations in joint contact during the critical early postnatal growth period may alter the course of hip development and may influence joint health in adulthood.

Increased body weight during later development and early adulthood has been associated with a greater risk of hip OA. Increased body weight after 2 mo of age in dogs has been associated with a more severe expression of DDH (13) and a greater prevalence of subsequent hip OA (14). A modest increase in body mass index at $18 \mathrm{y}$ in humans was associated with an increased risk of hip OA leading to joint replacement (15). However, the effect of increased body weight during early postnatal development, when congruent articulation is largely maintained by connective tissues, has not been investigated.

The objective of this study was to determine whether early postnatal body weight affected the course of hip development and the onset of OA in a canine model of DDH. We hypothesized that greater body weight during the first 3 wk after birth would result in an earlier age at ossification onset, reduced femoral head coverage at $4 \mathrm{mo}$, and greater probability of degenerative changes at $8 \mathrm{mo}$.

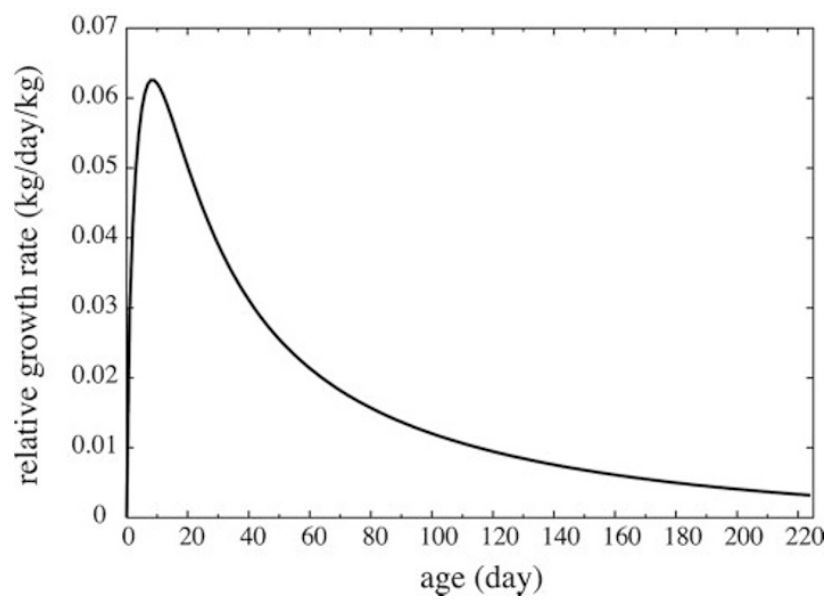

Figure 2. Rate of growth for body weight normalized by concurrent weight (study example).

\section{METHODS}

Animals. Labrador retrievers were obtained from a colony maintained by the James A. Baker Institute for Animal Health, Cornell University. Dogs were randomly selected from first available litters (phenotype of both sire and dam was either dysplastic or normal). The pattern of inheritance for hip morphology is complex. Dysplastic offspring from phenotypically normal parents, and vice versa, are common. Between 2 and 4 dogs were selected from 5 litters, resulting in a total of 17 subjects (Table 1).

Pups were introduced to solid food at $4 \mathrm{wk}$ and were weaned at $6 \mathrm{wk}$; feeding was ad libitum thereafter. Body weight was recorded daily for the first three weeks. Enclosures provided area for exercise. The Cornell University Institutional Animal Care and Use Committee approved all aspects of this study.

Radiography for onset of ossification. The pelvis was radiographed in a ventro-dorsal, hip-extended position (16). Radiographs were obtained every second day beginning at day 6 until ossification was observed in both femoral capital epiphyses. The age at which ossification was first observed for each femoral capital epiphysis was recorded as the age at ossification onset.

Radiography for femoral head coverage. The dorso-lateral subluxation procedure (17) was used to measure femoral head coverage at 4 mo. Dogs were anesthetized and placed in sternal recumbancy on a positioning pad with stifles flexed and femora in contact with and perpendicular to the table-top. Hips were in a neutral position and bore a portion of the body weight. The percentage of the femoral head area covered by the dorsal rim of the acetabulum was calculated from the dorso-ventral radiographic projection.

Necropsy evaluation and tissue collection. Subjects were euthanized at approximately 8 mo by barbiturate overdose. The articular cartilage collection procedure has been described previously (2). Soft tissues were dissected and the joint capsule was excised. The joint was evaluated for macroscopic fibrillation of articular cartilage and capsular fibrosis, and volumes of synovial fluid and ligament of the femoral head were measured. Cartilage was collected from the superior perifoveal region of the femoral head characteristic for early lesion formation. The entire lesion was collected as a single sample if present. Cartilage was also collected from the concentric surrounding area.

Biochemical assays. Biochemical assays were performed to detect the characteristic increase in water and fibronectin, and decrease in sulfated glycosaminoglycan (GAG) contents that precede macroscopic lesion formation $(2,18)$. Cartilage samples were lyophilized and weighed. Water content was expressed as a percentage of the weight at collection.

Dimethylmethylene Blue Assay: GAG content was measured by dimethylmethylene blue assay (19). 1,9-dimethyl-methylene blue (all chemicals: Sigma-Aldrich, Inc., St. Louis, MO, USA), a strongly metachromatic dye, was added to papain digested samples and the absorbance of the GAG-dye complex was measured at $535 \mathrm{~nm}$ on a spectrophotometer (Beckman Instruments, Inc., Fullerton, CA, USA). The standard was a serial dilution of chondroitin-4 sulfate.

Fibronectin ELISA: Fibronectin content was measured by ELISA (20). Urea extracted samples were assayed with a sandwich ELISA. The capture and detector antibodies were goat anti-human fibronectin (all antibodies: ICN/Cappel, Aurora, OH, USA) and rabbit anti-human fibronectin, respectively. Antigen-detector antibody complexes were quantified using a peroxidase-linked goat anti-rabbit IgG antibody and an O-phenylenediamine substrate. Absorbance was measured at $490 \mathrm{~nm}$ with a reference wavelength of $630 \mathrm{~nm}$ on a microtitre plate reader (Tecan, Research Triangle Park, NC, USA). The standard was a serial dilution of purified plasma fibronectin.

Degeneration score assignment. A degeneration score of 0 to 3 was assigned to each hip based on cartilage biochemistry and necropsy examination (Table 2). Constituent values from the superior perifoveal area characteristic for lesion formation and the surrounding area were used for score assignment. Changes in gross joint structure characteristic of degeneration associated with DDH include capsular fibrosis, hypertrophy of the femoral head ligament, effusion, and macroscopic cartilage fibrillation. Hips were scored independently by two of the authors (W.S.V.B.-F., R.J.T.). Discrepancies were reviewed and a score was reached by consensus.

Table 1. Sample summary

\begin{tabular}{cclc}
\hline Parental phenotype & Litter & \multicolumn{1}{c}{ Gender } & Litter size \\
\hline Dysplastic & 1 & 2 female & 8 \\
& 2 & 1 female, 2 male & 8 \\
\multirow{2}{*}{ Normal } & 3 & 2 female, 2 male & 9 \\
& 4 & 2 female, 2 male & 12 \\
& 5 & 2 female, 2 male & 7 \\
\hline
\end{tabular}


Table 2. Degeneration score assignment guidelines

\begin{tabular}{|c|c|c|c|}
\hline Score & Necropsy observation & Cartilage 1 & \\
\hline 0 & No abnormalities & Within normal range* & \\
\hline 1 & $\begin{array}{l}\text { Mild changes: ligament hypertrophy (volume }<1 \mathrm{ml} \text { ), mild capsular } \\
\text { fibrosis, minimal effusion, no cartilage fibrillation }\end{array}$ & $\begin{array}{l}\text { Water increase } \\
\text { GAG }^{* *} \text { decrease } \\
\text { Fibronectin }\end{array}$ & $\begin{array}{l}<5 \% \\
<20 \% \\
<600 \mathrm{ng} / \mathrm{mg}\end{array}$ \\
\hline 2 & $\begin{array}{l}\text { Moderate changes: ligament hypertrophy (volume }>1 \mathrm{ml} \text { ), } \\
\text { extensive capsular fibrosis, effusion, no cartilage fibrillation }\end{array}$ & $\begin{array}{l}\text { Water increase } \\
\text { GAG decrease } \\
\text { Fibronectin }\end{array}$ & $\begin{array}{l}>5 \% \\
>20 \% \\
>600 \mathrm{ng} / \mathrm{mg}\end{array}$ \\
\hline 3 & Macroscopic lesion present & & \\
\hline
\end{tabular}

* Reference values based on (2).

** Expressed per mg cartilage dry weight.

Statistical analyses. Data in this study occur in a natural hierarchical structure, with hips nested within dogs and dogs nested within litters. Observations are not independent, as hips within a dog are expected to be more similar than hips from different dogs, and dogs from the same litter are expected to be more similar than dogs from different litters. Hierarchical linear models (HLMs) explicitly address data dependency by using a submodel for each level of the hierarchy; each submodel includes a level-specific error term (21).

A key feature of HLMs is that the total variance of the response variable is decomposed into components contributed by individual levels. A representative 2-level random effects model for the observed growth rate contained the grand mean, $\gamma_{00}$, a litter-level error (random) term $u_{0 j}$, and a dog-within-litter error (random) term $r_{i j}$; the growth rate for $\operatorname{dog} i$ in litter $j, \lambda_{i j}$, was

$$
\lambda_{i j}=\gamma_{00}+u_{0 j}+r_{i j}
$$

Variance of the growth rate was

$$
\operatorname{var}\left(\lambda_{i j}\right)=\operatorname{var}\left(u_{0 j}\right)+\operatorname{var}\left(r_{i j}\right)=\sigma_{\text {litter }}^{2}+\sigma_{\text {dog }}^{2}
$$

Variance components were expressed as intraclass correlations, a measure of within-group similarity, for 2-level models (22) or as a percentage of the total variance for 3-level models. HLMs used included both fixed effects (phenotype, gender) and random effects (litter, dog within litter, hip within dog). See (23) for a review of effect classification in mixed models.

The 8-mo hip degeneration score was an ordinal variable. A proportional odds model was used to estimate the effect of the independent variable on the $\log$ odds of a particular degeneration score (24). A typical model was

$$
\operatorname{logit}[P(\text { score } \leq j)]=\alpha_{j}+\beta w, j=1, \ldots, J-1
$$

The model assumes that the effect of the independent variable body weight, $w$, is the same (proportional) for all degeneration score categories. The probability of a hip degeneration score indicating moderate biochemical changes (score 2) or lesion (score 3) was estimated from model results.

HLMs were implemented using the MIXED procedure in the SAS statistical software package (version 9.1, SAS Institute, Cary, NC). The restricted maximum likelihood procedure was used to estimate variance components. Degrees of freedom for F statistics were estimated using the Kenward-Roger approximation for unbalanced data (25). Estimated degrees of freedom are often fractional values. Proportional odds models were implemented using the LOGISTIC procedure in SAS. Rate of growth for the first three weeks of age was estimated using a simple exponential growth model $\left(W=W_{0} e^{\lambda t}\right)$. Strength of the linear relationship between body weight at successive ages and onset of ossification was estimated using ordinary least squares.

Statistical analysis consisted of three stages. First, the pattern of growth (body weight gain) for days $0-20$ was examined. Next, the ability of the growth parameters to predict the age at ossification onset for the femoral capital epiphyses was investigated. Finally, the ability of the early growth parameters to predict outcomes of femoral head coverage and degeneration at early adulthood was investigated. $p$-values below 0.05 were considered statistically significant.

\section{RESULTS}

Body weight for 0-20 d. Mean body weight at birth was $0.44 \mathrm{~kg}$ (SE 0.04). All pups were within two standard deviations of the colony mean $(\mu 0.43, \sigma 0.08 \mathrm{~kg})$. Of the total variance in birth weight, $85.1 \%$ was due to among-litter differences and $14.9 \%$ to within-litter differences. Litter size was strongly associated with birth weight $(F(1,2.77)=39.37$, $p=0.010$ ), accounting for $94.7 \%$ of the among-litter variance; pups from larger litters had lower birth weights. Birth weight for females was less than that for males (by $19.8 \mathrm{~g}$ ); however, the difference was not significant $(F(1,12.9)=1.41$, $p=0.256)$ when controlling for litter size. There was no difference in birth weight between normal and dysplastic parental phenotypes $(F(1,1.85)=0.54, p=0.543)$ when controlling for litter size. The body weight growth trajectory for the first $20 \mathrm{~d}$ was approximately exponential (Fig. 3). Mean exponential growth rate (EGR) for body weight was $0.0641 / \mathrm{d}$ (SE 0.004). EGR for females was less than that for males (by 0.007) $(F(1,11.4)=8.74, p=0.013$ ) when controlling for birth weight. An intraclass correlation of $71.3 \%$ indicated the EGR was more similar within litters than among litters. Birth weight prediction of EGR approached conventional levels of significance $(F(1,11.5)=3.69, p=$ 0.080 ), with lower birth weight subjects having a greater EGR. Inclusion of birth weight in the model reduced among-litter variance by $25.5 \%$ and within-litter variance by $20.1 \%$. There was no difference in EGR between normal and dysplastic parental phenotypes $(F(1,1.9)=0.27, p=0.657)$. The percentage of the total variance in body weight explained by litter size decreased from $82.7 \%$ at day 0 (birth) to $32.8 \%$ at age $8 \mathrm{~d}$. By age $12 \mathrm{~d}$, the association between litter size and body weight was no longer significant.

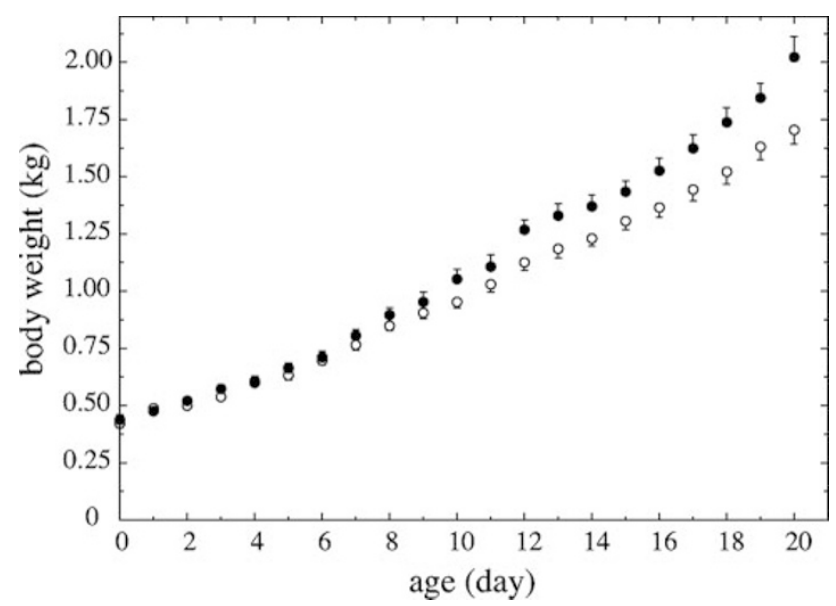

Figure 3. Body weight means for males $(\bigcirc)$ and females $(\bigcirc)$ from 0 to $20 \mathrm{~d}$ ( $n=9$ female, $n=8$ male). Error bars represent SE. 
Age at femoral head ossification onset. Mean age at ossification onset was $12.12 \mathrm{~d}$ (SE 0.99). All pups were within two standard deviations of the colony mean $(\mu 12.69, \sigma 3.72 \mathrm{~d})$. Of the total variance in age at ossification onset, $68.8 \%$ was due to among-litter differences, $23.4 \%$ to within-litter differences, and $7.8 \%$ to between-hip differences. Age at ossification onset was strongly associated with birth weight $(F(2,30)=$ $38.27, p<0.001$ ), with lower birth weight pups having a later age at onset. There was a significant interaction between parental phenotype and birth weight $(F(1,12)=5.14, p=0.043)$, with pups of normal parental phenotype having a slope of greater magnitude (Fig. 4). Inclusion of the interaction in the model reduced the within-litter variance by $22.8 \%$. The slope for the dysplastic parental phenotype was not significantly different from zero $(t(12)=-1.64, p=0.126)$. Ossification onset occurred $1.5 \mathrm{~d}$ earlier for females at the birth weight mean $(F(1,12)=$ $9.43, p=0.010)$. The percentage of the total variance in age at ossification onset explained by body weight decreased rapidly from $65 \%$ at day 0 (birth) to $17 \%$ at age $8 \mathrm{~d}$ (Fig. 5). (The strength of the initial relationship would persist if all subjects had identical growth rates.) Inclusion of body weight in the model did not reduce within-litter variance at any age before the age at ossification onset. Age at ossification onset was not significantly associated with the EGR $(F(1,14)=0.24, p=0.632)$.

Four-month femoral head coverage. Mean femoral head coverage was $64.0 \%$ (SE 1.9). No dislocations were detected radiographically or during physical exams. Femoral head coverage was associated with birth weight $(F(1,15)=6.59, p=$ $0.021)$ and day 1 weight $(F(1,15)=4.59, p=0.049)$; lower weight subjects had greater femoral head coverage (Fig. 6). Of the total variance in femoral head coverage, $4.5 \%$ was due to among-litter differences, $35.3 \%$ to within-litter differences, and $60.3 \%$ to between-hip differences. Inclusion of birth weight in the model reduced within-litter variance by $40.0 \%$ and accounted for all of the among-litter variance. Femoral head coverage was not associated with parental phenotype $(F(1,3.12)=0.00, p=0.955)$.

Eight-month joint degeneration score. At $8 \mathrm{mo}, 9$ hips had no abnormalities, 7 hips were mildly affected, 12 hips were

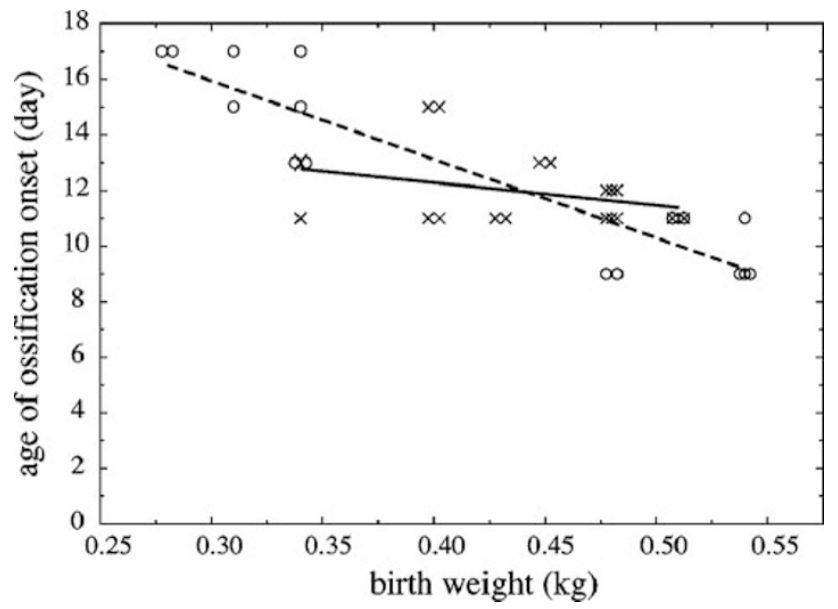

Figure 4. Age at femoral head ossification onset for pups from normal $(\bigcirc$, - - ) and dysplastic ( $\times,-$ ) parental phenotypes ( $n=34$ hips; $n=16$ normal, $n=18$ dysplastic phenotype). Coincident data points were moved by $0.0025 \mathrm{~kg}$ increments $(\Delta)$ to improve clarity ( 2 points: $-\Delta,+\Delta ; 3$ points: $-\Delta, 0,+\Delta$ ).

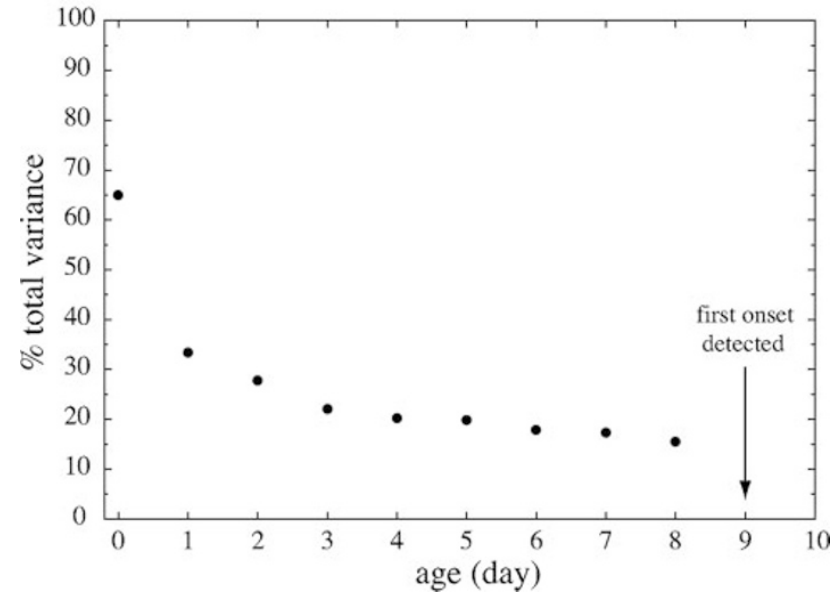

Figure 5. Percent of total variance for age at ossification onset explained by body weight for each age ( $n=34$ hips).

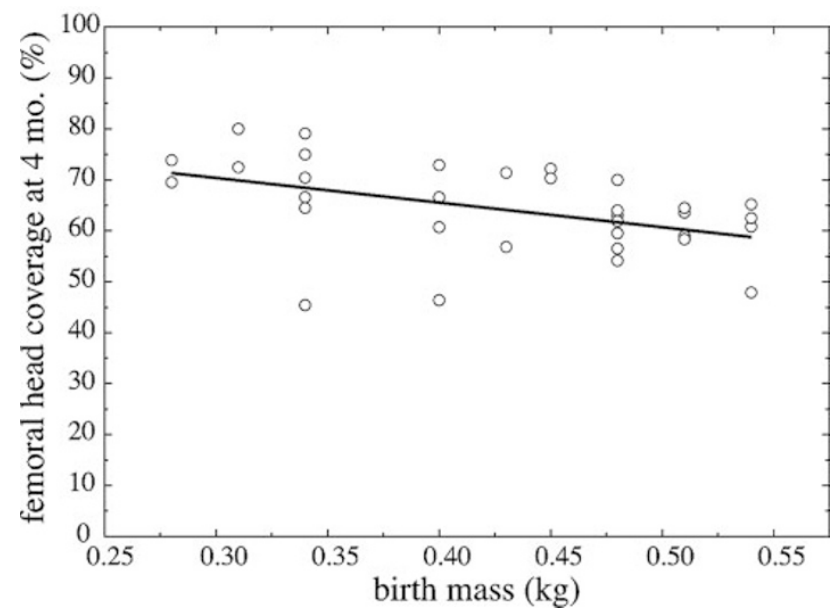

Figure 6. Femoral head coverage at 4 mo as a function of birth weight $(n=$ 34 hips). Solid line represents the hierarchical linear model.

moderately affected, and 6 hips had a macroscopic lesion (Table 3, Fig. 7 example). Right and left hips were affected similarly with a maximum score difference of 1 . The probability of moderate cartilage abnormality or lesion increased with increasing birth weight $\left(\chi^{2}=6.24, p=0.013\right)$ and was lower for females than for males $\left(\chi^{2}=4.08, p=0.043\right)$ (Fig. 8). This probability increased with decreasing 4 mo femoral head coverage $\left(\chi^{2}=11.93, p<0.001\right)$. The probability was not associated with parental phenotype $\left(\chi^{2}=0.51, p=0.477\right)$.

\section{DISCUSSION}

Much research has focused on cartilage degeneration secondary to DDH (1-3); however, little is known about influences such as body weight on early postnatal hip development. The objective of this initial study was to determine whether early postnatal body weight affected the course of hip development and joint health at adulthood in a canine model of DDH.

When studying early postnatal skeletal growth, prenatal growth and skeletal maturity at birth must be considered. Prenatal growth is the result of the interaction between the fetal genotype and the maternal intrauterine environment. One way in which the intrauterine environment influences growth is through a uterine size constraint during late gestation. The 
Table 3. Summary of cartilage extracellular matrix constituents and femoral head coverage by degeneration score group

\begin{tabular}{ccccc}
\hline Score & Water $(\%)$ & GAG* $(\mu \mathrm{g} / \mathrm{mg})$ & Fibronectin* $\dagger(\mathrm{ng} / \mathrm{mg})$ & Femoral head coverage $(\%)$ \\
\hline 0 or 1 & $74.9(0.77)$ & $136.6(6.99)$ & $317.4[251.4,465.0]$ & $68.1(1.7)$ \\
2 or 3 & $78.1(0.78)$ & $115.5(4.73)$ & $958.4[691.3,1221.1]$ & $60.5(2.0)$ \\
\hline
\end{tabular}

* Expressed per mg tissue dry weight.

$\dagger$ Data summarized as median [1st, 3rd quartiles]; all other data summarized as mean (SE).
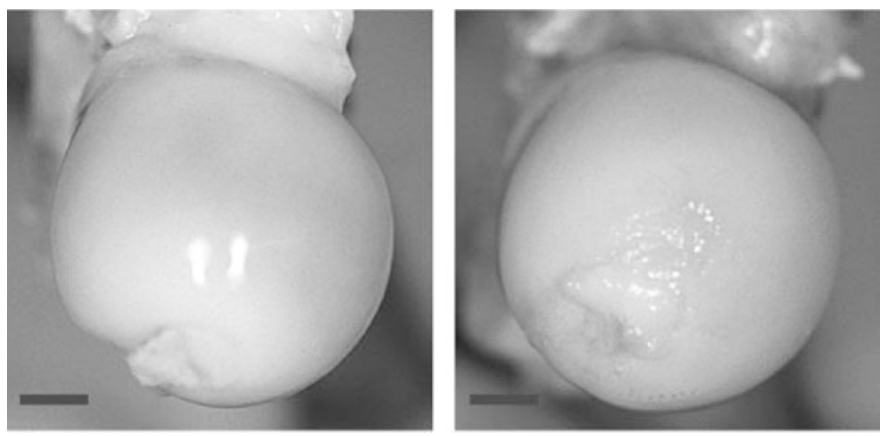

Figure 7. Articular surface for normal (L) and lesion (R) femoral heads. Note roughness in lesion example. $\mathrm{Bar}=5 \mathrm{~mm}$.

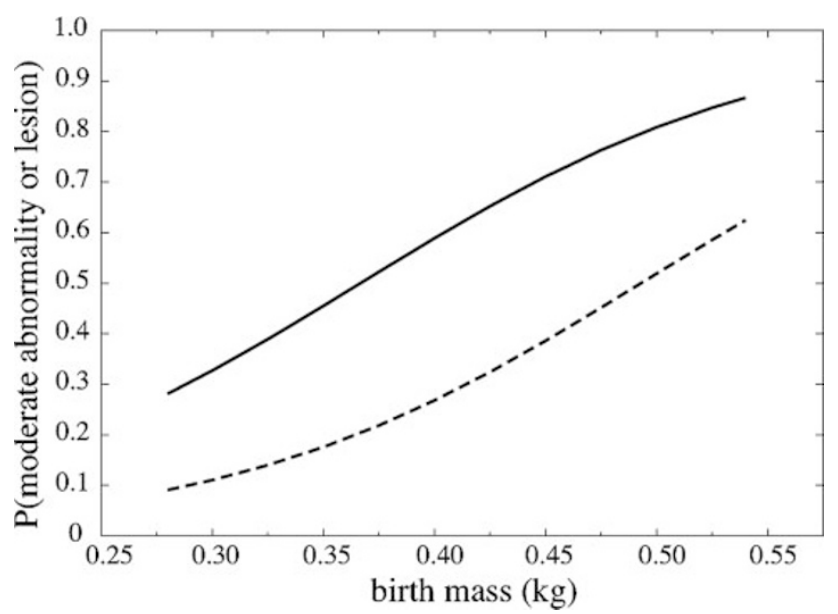

Figure 8. Probability of moderate abnormality or lesion of femoral head cartilage for males (-) and females (- - ) at $8 \mathrm{mo}(n=9$ female, $n=8$ male).

uterine size constraint results in a negative association between the number of fetuses and birth weight. This effect is seen in humans, where fetal weight for singletons and twins is similar until approximately $28 \mathrm{wk}$, after which the rate of growth for twins slows relative to that of singletons (26). A similar effect is seen in mice, where the negative correlation of litter size and fetal weight does not begin until day 17.5 in a $19 \mathrm{~d}$ gestation (27). After birth, a period of accelerated, or catch-up, growth occurs (28). A negative association between litter size and birth weight was also found in this study. Lower birth weight pups had a greater EGR, indicating that postnatal catch-up growth occurred. Intrauterine growth restriction was likely greater for larger litters.

If ossification rate is positively associated with volumetric growth rate, pups from larger litters would be skeletally less mature (proportionately less ossification) at birth than pups from smaller litters. There is little research addressing the relationship between birth weight and the extent of skeletal ossification. In late term pregnancy for rats (day 21), the extent of ossification in skeletal structures where ossification is beginning or still in progress is positively related to fetal weight (29). If this result in rats represents a general phenomenon of mammalian prenatal development, then the lower birth weight pups in the present study can be expected to have proportionately less ossification of the acetabulum and proximal femur at birth.

Skeletal maturity at birth is also determined by length of gestation. Gestation length in litters of five or more pups has been shown to be shorter by $1.1 \mathrm{~d}$ than litters with fewer than 5 pups (30). Any decrease in length of gestation for larger litters would be a minor additive influence to the effects of late term growth rate reduction.

The age at ossification onset was significantly associated with birth weight; however, the variance attributed to body weight decayed rapidly with increasing age. This result is consistent with the degree of skeletal maturity at birth being the primary determinant of age at onset rather than body weight per se. Inclusion of body weight in the model did not reduce within-litter variance in age at ossification onset. A delay in skeletal maturity due to intrauterine crowding is a feature common to all pups within a litter. The remaining variance attributed to body weight at days 1 to 8 is likely due to the residual correlation between body weight and weight at birth.

The interpretation that degree of skeletal maturity at birth was the primary determinant of age at ossification onset does not imply that mechanical forces were not important for stimulating the onset of ossification. In clear cases of unilateral $\mathrm{DDH}$, the onset and progression of ossification in the affected hip are delayed relative to the normal hip (31). The normal hip serves as a control for environmental and systemic factors, so the response due to differences in local mechanical environmental variables can be distinguished from other influences. This result supports the claim by Wilkinson (31) that a delay in the onset of ossification for the femoral epiphyses (up to age 12 mo in human infants) was "of little consequence"; however, a unilateral retardation of ossification "might well suggest some pathological condition."

The interaction between birth weight and parental phenotype was significant when gender differences and birth weight were controlled. The magnitude of the slope for pups from dysplastic parental phenotype was less than the slope for pups from normal parental phenotype. The variance accounted for by the interaction was at the within-litter level. DDH in the Labrador retriever model is associated with greater hip joint laxity (9). If laxity is present at birth, the femoral head for pups with a dysplastic parental phenotype may be loaded in a more focal, eccentric manner, thus altering the stimulus for ossification onset. Parental phenotype, however, was not predictive of 4 mo femoral head coverage or 8 mo degeneration 
score. The interpretation of an interaction for a small sample size should be made with considerable caution. Existence of this interaction must be confirmed in a larger sample.

Greater birth and day 1 weights were associated with reduced femoral head coverage at 4 mo. Birth weight reduced the within-litter variance by $40.0 \%$. Greater birth weight may exceed the capacity of the immature connective tissues to maintain fully congruent articulation between the femoral head and the acetabulum. A subluxated femoral head will result in a smaller contact area and thus greater contact stresses on the dorsal lateral margin of the acetabulum (32). This result suggests that birth weight was an important factor in establishing the manner in which the femoral head and acetabulum articulated, and as a result, influenced the development of acetabular depth.

The probability of moderate biochemical changes or lesion of the femoral head cartilage increased with increasing birth weight. The probability of degenerative changes for a given birth weight was lower for females than for males. There is no clear gender difference in DDH incidence for dogs as there is for humans (9). Persistence of a gender difference would be unlikely in a larger study population. The increased probability of degenerative changes with decreased femoral head coverage was similar to previous findings in Labrador retrievers (33). Degenerative changes were located in the characteristic superior perifoveal region of the femoral head that articulates with the dorsal rim of the acetabulum in subluxated hips (2). The degenerative changes are likely due to elevated contact stress that is a result of the focal, eccentric loading (34).

The lack of association between parental phenotype and 4 mo femoral head coverage and 8 mo degeneration score was likely due to the small sample size. Dysplastic progeny from phenotypically normal matings, and vice versa, are common. With this inheritance pattern, it is unlikely that parental phenotype will be strongly predictive of offspring phenotype in a small sample.

In conclusion, this study shows that increased early postnatal body weight, particularly birth weight, was associated with reduced femoral head coverage at 4 mo and a greater probability of degenerative changes at $8 \mathrm{mo}$. Increased birth weight was negatively associated with age at ossification onset for the femoral head; however, the effect was likely due to differences in skeletal maturity rather than to weight per se. These results support the hypothesis that increased body weight during the critical early postnatal period was sufficient to alter the course of hip development and result in measurable degenerative changes at adulthood.

Acknowledgments. The authors thank Alma Jo Williams for animal care and radiography, Margaret Vernier-Singer for assistance with tissue collection and biochemical assays, Liz Corey for performing the fibronectin ELISAs, and the James A. Baker Institute for Animal Health, Cornell University, for access to the Labrador retriever breeding colony.

\section{REFERENCES}

1. Weinstein SL 1987 Natural history of congenital hip dislocation (CDH) and hip dysplasia. Clin Orthop Relat Res 225:62-76
2. Burton-Wurster N, Farese JP, Todhunter RJ, Lust G 1999 Site-specific variation in femoral head cartilage composition in dogs at high and low risk for development of osteoarthritis: insights into cartilage degeneration. Osteoarthritis Cartilage 7:486-497

3. Jacobsen S, Sonne-Holm S, Søballe K, Gebuhr P, Lund B 2005 Hip dysplasia and osteoarthritis a survey of 4151 subjects from the Osteoarthrosis Substudy of the Copenhagen City Heart Study. Acta Orthop 76:149-158

4. Committee on Quality Improvement, Subcommittee on Developmental Dysplasia of the Hip 2000 Clinical practice guideline: early detection of developmental dysplasia of the hip. Pediatrics 105:896-905

5. Riser WH 1975 The dog as a model for the study of hip dysplasia. Growth, form, and development of the normal and dysplastic hip joint. Vet Pathol 12:234-334

6. Cunningham FG, MacDonald PC, Gant NF 1989 Williams Obstetrics. Appleton \& Lange, Norwalk, pp 177-180

7. Walker JM, Goldsmith CH 1981 Morphometric study of the fetal development of the human hip joint: significance for congenital hip disease. Yale J Biol Med 54:411-437

8. Breur GJ, Lust G, Todhunter RJ 2001 Genetics of canine hip dysplasia and other orthopaedic traits. In: Ruvinsky A, Sampson J (eds) The Genetics of the Dog. CABI Publishing, New York, pp 268-277

9. Lust G 1993 Other orthopedic diseases: hip dysplasia in dogs. In: Slatter D (ed) Textbook of Small Animal Surgery. WB Saunders Company, Philadelphia, pp 19381944

10. Schoenecker PL, Lesker PA, Ogata K 1984 A dynamic model of experimental hip dysplasia - gross and histological pathology, and the effect of position of immobilization on capital femoral epiphyseal blood-flow. J Bone Joint Surg Am 66:12811288

11. Harrison TJ 1961 The influence of the femoral head on pelvic growth and acetabular form in the rat. J Anat 95:12-26

12. Coleman CR, Slager RF, Smith WS 1958 The effect of environmental influence on acetabular development. Surg Forum 9:775-780

13. Kealy RD, Olsson SE, Monti KL, Lawler DF, Biery DN, Helms RW, Lust G, Smith GK 1992 Effects of limited food consumption on the incidence of hip dysplasia in growing dogs. J Am Vet Med Assoc 201:857-863

14. Kealy RD, Lawler DF, Ballam JM, Lust G, Biery DN, Smith GK, Mantz SL 2000 Evaluation of the effect of limited food consumption on radiographic evidence of osteoarthritis in dogs. J Am Vet Med Assoc 217:1678-1680

15. Karlson EW, Mandl LA, Aweh GN, Sangha O, Miang MH, Grodstein F 2003 Total hip replacement due to osteoarthritis: the importance of age, obesity, and other modifiable risk factors. Am J Med 114:93-98

16. Todhunter RJ, Zachos TA, Gilbert RO, Erb HN, Williams AJ, Burton-Wurster N, Lust G 1997 Onset of epiphyseal mineralization and growth plate closure in radiographically normal and dysplastic Labrador retrievers. J Am Vet Med Assoc 210:1458-1462

17. Farese JP, Todhunter RJ, Lust G, Williams AJ, Dykes NL 1998 Dorsolateral subluxation of hip joints in dogs measured in a weight-bearing position with radiography and computed tomography. Vet Surg 27:393-405

18. Mankin HJ, Brandt KD 1997 Pathogenesis of osteoarthritis. In: Kelley WN, Ruddy S, Harris ED, Sledge CB (eds) Textbook of Rheumatology. WB Saunders Company, Philadelphia, pp 1369-1382

19. Farndale RW, Sayers CA, Barrett AJ 1982 A direct spectrophotometric microassay for sulfated glycosaminoglycans in cartilage cultures. Connect Tissue Res 9:247-248

20. Wurster NB, Lust G 1982 Fibronectin in osteoarthritic canine articular cartilage. Biochem Biophys Res Commun 109:1094-1101

21. Raudenbush SW, Bryk AS 2002 Hierarchical Linear Models: Applications and Data Analysis Methods, ed 2. Sage, Thousand Oaks, pp 228-251

22. Kuehl RO 2000 Design of Experiments: Statistical Principles of Research Design and Analysis, ed 2. Duxbury, Pacific Grove, pp 148-169

23. Searle SR, Casella G, McCulloch CE 1992 Variance Components. John Wiley \& Sons, New York, pp 1-18

24. Agresti A 2002 Categorical Data Analysis. JohnWiley \& Sons, Hoboken, pp 274-282

25. Littell RC 2002 Analysis of unbalanced mixed model data: a case study comparison of ANOVA versus REML/GLS. J Agric Biol Environ Stat 7:472-490

26. Snow MH 1986 Control of embryonic growth rate and fetal size in mammals. In: Faulkner F, Tanner JM (eds) Human Growth A Comprehensive Treatise. Plenum Press, New York, pp 67-82

27. Snow MH 1989 Embryonic growth and the experimental manipulation of fetal size In: Tanner JM, Preece MA (eds) The Physiology of Human Growth. Cambridge University Press, Cambridge, pp 1-10

28. Harrison GA, Tanner JM, Pilbeam DR, Baker PT 1988 Human Biology. Oxford University Press, New York, pp 339-360

29. Chahoud I, Paumgartten FJ 2005 Relationships between fetal body weight of Wistar rats at term and the extent of skeletal ossification. Braz J Med Biol Res 38:565-575

30. Eilts BE, Davidson AP, Hosgood G, Paccamonti DL, Baker DG 2005 Factors affecting gestation duration in the bitch. Theriogenology 64:242-251

31. Wilkinson JA 1985 Congenital Displacement of the Hip Joint. Springer-Verlag, New York, pp 59-85

32. Pompe B, Daniel M, Sochor M, Vengust R, Kralj-Iglič V, Iglič A 2003 Gradient of contact stress in normal and dysplastic human hips. Med Eng Phys 25:379-385

33. Lust G, Williams AJ, Burton-Wurster N, Pijanowski GJ, Beck KA, Rubin G, Smith GK 1993 Joint laxity and its association with hip dysplasia in Labrador retrievers. Am J Vet Res 54:1990-1999

34. Mavčič B, Pompe B, Antolič V, Daniel M, Iglič A, Kralj-Iglič V 2002 Mathematical estimation of stress distribution in normal and dysplastic human hips. J Orthop Res 20:1025-1030 